\title{
Application of PLC in automatic control system in the production of steel
}

\author{
FAN Zhechao, FENG Hongwei \\ Inner Mongolia Technical College of Mechanics and Electrics, 010070,China \\ fanzhechao83@sina.com
}

Key words : PLC; automatic control system; control;

\begin{abstract}
The PLC control system is used to control and adjust the parameters in the process of high strength steel production, and the working principle and process of PLC control is introduced. Experience of production practice shows that the system has a strong operational and practical, can greatly improve the production efficiency and product quality.
\end{abstract}

\section{Introduction}

With the development of the construction industry, the construction steel production has been developed rapidly. Application of electrical automation technology in metallurgical industry with the rapid development of automation technology, as well as the urgent need to improve production quality and production efficiency of the metallurgical industry, the application scope and application of the steel industry has increased year by year. The automation level of iron and steel industry is not only a symbol of industrial modernization, but also can make the enterprise obtain huge economic benefits. However, it is known that the automation control of high strength heat treatment production line is less than [1], with the rapid development of automation technology, the automatic control of the whole process of high strength steel production line can not only improve the quality of steel products, but also improve the efficiency of production, save the capital, improve the economy, and promote the rapid development of steel industry.

\section{Overview}

\section{PLC control system}

PLC is a kind of memory that can be programmed to store operations in its internal memory, and can through digital or analog input and output, control various types of machinery or production process. PLC and related peripheral equipment should be in accordance with the easy and industrial control system to form a whole, easy to expand its function principle and design.

PLC device sub and modem, the bureau is responsible for communication with internal PLC modem and the connection with the external network. In communication, the data from the user into the modem modulation, transmission through the user's power distribution lines to the end of the device, the end of the signal demodulation, and then go to the external Internet. The operation of the PLC control system mainly includes the input sampling stage, the program execution stage and the output refresh stage, and the three stages of the operation process of the [2]. During the sampling period, the PLC reads all the input signals into the input signal and the input state is stored in the corresponding input image register. In the subsequent program execution stage and output refresh stage, the input image register signal with the outside world, until the next scan cycle of the new signal, will be re - save. Visible, PLC in the implementation process and processing data, not directly using the input signal on the scene, and the use of this sampling input to the image area data. 
In the program execution phase, PLC according to the ladder diagram program, from left to right, from top to bottom step by step process, when the instruction involved in the input, output state, PLC input from the input image register "read" corresponding input terminal state, from the output image of the "read" corresponding element ("soft relay") of the current state. In the output refresh stage, the operation results of the program execution stage are stored in the output image, and not to the output port. The PLC outputs the output variable into the output latch, and then generates the control output of the cycle by the latch. If the state of the internal output relay is "1", the output relay contacts are closed, and the output terminal is driven by an external load. These three stages are completed for a complete scan cycle.

\section{the working principle and process flow of high strength steel}

High strength steel production line control system mainly includes the power control system (mainly used to control the temperature and time of quenching), cooling medium control system (control cooling speed, etc.), micro tension system (mainly used to control the flatness and relaxation performance), etc.. PLC control system of this paper uses S7-400PLC Siemens products, through the DP network and remote I/O station and inverter communication, to achieve the entire production line control [4,5]. Among them, the core components of the central processing unit 414-2DP.

High strength heat treatment production process of steel bar production line as the main, wire rod on the line, and line, a mechanical to rust, drawing forming, straightening straight, No. 1, No. 2, drawing, hydraulic shear, reversing, rewinding, packaging and storage, induction heating quenching, water cooling, induction tempering, water cooling, drawing. During heat treatment of high strength steel, temperature, cooling speed and tension are the key parameters of the whole production line. The whole line is controlled by PLC, and the temperature and tension can be adjusted, and the whole production line can be quickly and efficiently. The production line is made up of [1], such as the wire drawing machine, the straightening machine, the traction machine, the quenching induction heating furnace, the spray cooler, the tempering induction heating furnace, the straightening machine, the vertical collection and so on. The appropriate tension between the receiving line and the traction machine can be formed, and the feedback of the tension measuring instrument to the PLC system is given. In the production process of high strength steel, the quenching temperature is usually selected from 850 to 1040 , and the tempering temperature is generally selected from 400 to 600 , and the cooling medium is water, and the temperature of quenching and tempering is measured by infrared temperature measurement system. The PLC system can be adjusted in real time.

\section{Application of PLC based automatic control system in high strength steel production}

\section{PLC application}

(1) temperature control

Quenching temperature and tempering temperature are the two most important parameters in heat treatment process, which need to be controlled accurately and stably. PLC according to the actual temperature of the infrared thermometer feedback and process set temperature value for comparison, get the deviation. According to the deviation of the PID algorithm, the heating power of induction heating furnace is adjusted to realize the closed-loop control of steel bar. In most cases, the end voltage of the inductor is fixed in design and practical use, and the voltage is constant from the beginning to the end of the heating. Only in the cycle of induction heating, when the blank heating requires temperature only need to reduce voltage, or is in induction heating magnetic materials hesitate on heating above the Curie point, the disappearance of magnetic material, heating speed slows down, in order to improve the heating speed and improve the terminal voltage of the 
induction machine. When the heating temperature of the steel bar is more stringent, it should be used to stabilize the power supply voltage, so the power supply system on the need to increase the power supply system, to ensure that the sensor's end voltage fluctuations in $2 \%$, which is used in the heat treatment of workpiece heat treatment is very important, otherwise it will cause a long workpiece heat treatment mechanical properties are not consistent with [6]. Therefore, in the production process of high strength steel, in order to make the heating temperature of the production line is stable, the power control of the induction furnace adopts constant power design, the constant power control can make the production line power is not affected by the power supply system.

(2) control of cooling rate

Cooling rate is one of the most important parameters in the process of heat treatment, and most of the temperature variation range of most of the quenching process is from 25 to 35 . Through the PLC system can be more accurate control of the cooling rate, the actual production process, the cooling water temperature in the continuous changes, PLC system can be used to adjust the online feedback data. For tap water spray cooling performance of test parameters as the main nozzle pressure and flow characteristic curve, a nozzle injection angle, nozzle minimum and maximum flow value, spray flow density, cooling time etc.. The characteristics of the combination of the nozzle pressure, flow characteristics, spray angle, can reflect the spray characteristics of the spray nozzle, flow regulation range, etc.. Production of different specifications of steel products, the system automatically call the database in the flow and pressure experience value feedback to the PLC system, which automatically adjust the flow of the electric flow control valve opening, the system will automatically adjust the flow into the database.

(3) tension control

Tension control is the most important link in the tension control, and the effect of tension control is directly related to the quality of the finished product. The purpose of tension control is to ensure that the tension of the tension in the heat treatment area is constant at the set value, so as to ensure the flatness and mechanical properties of the finished product. In order to accurately control the tension of heat treatment, the torque of the motor must be accurately controlled in order to accurately control the tension. By the speed control to tension control when the speed of a given additional set of a saturation value, so that it is greater than the actual speed, at this time the speed regulator output saturation. Tension is given by the tension wheel radius plus dynamic compensation, no-load compensation, friction torque compensation and bending torque compensation that is given, as the winding machine torque / current limit. Because the output of the speed regulator reaches the saturation limit, the torque limit can be controlled to control the torque of the motor and ensure the tension constant.

\section{system analysis and design}

(1) variable frequency speed control mode

For the traction machine and the first round, the process requires starting to stop quickly, running smoothly, and the speed adjustment precision and torque control have higher requirements. Frequency converter control mode is the key to determine the practical performance of variable frequency power supply. It is divided into two kinds of non intelligent control and intelligent control. Vector control as a typical representative of the non intelligent control, it is the essence of the speed and the magnetic field for independent control. Using the vector control mode of the inverter power supply, the speed range is large, can start from zero speed control, but also can control the torque, the corresponding speed of the system is also faster, but also can realize the asynchronous motor torque control. In this paper, the vector control method is improved, and the automatic detection and automatic adjustment function is realized on the parameters of the drive induction motor. Therefore, 
this paper adopts vector control method with the encoder to speed regulation, achieve the closed-loop control of speed, is a kind of high strength steel production line production process control mode.

(2) communication between PLC and inverter

From the PLC to the control of each inverter, speed setting, as well as the actual value of the power transmission to the PLC and the actual value of the operation state signal to transmit, is the transmission system design to face a choice, with the use of basic automation system and frequency conversion device has a strong communication function, the use of communication mode for data transmission is a reliable and practical application, and increasingly popular program, compared to hard wiring way to save control cable.

This system adopts Profibus-DP bus communication technology, which is widely used in the industry. For this purpose, each converter is equipped with CBP2 serial communication board, and the physical connection between the inverter and PLC is realized by anti jamming performance good twisted pair cable and bus connector. On the Profibus-DP bus, the PLC and the transmission device have a unique communication address on the bus. The communication speed is set up with the parameters of the transmission medium and the bus length. The communication speed of the system is set to $187.5 \mathrm{~Kb} / \mathrm{s}$. The data in the bus is transmitted periodically in the form of packets. The packets contain process data area (PZD) and the PKW (PKW). PZD is expressed as the content of the control word and the actual value.

Based on the consideration of production safety, the relevant "emergency stop" signal is transmitted to the CUVC control board of DI control board through hard wiring, and the corresponding parameters are set up.

\section{conclusion}

The successful application of PLC control system in the production line of high strength steel will greatly improve the quality and production efficiency of domestic high strength steel products, which has a significant effect on the control field and the technological progress of the metallurgical field. PLC control system used in this paper, the whole process of high strength steel production line, and has been successfully applied to the domestic high strength steel production base, according to customer feedback information shows that the use of PLC control system for the overall design of production lines, production lines running stable, control flexible, accurate, saving production costs, based on the system of production line control system is expected to spread across the country.

\section{Reference}

[1] Fan Meiguang, Yang Ju Xing. High strength reinforced heat treatment production line automatic control system [J]. Heat treatment technology and equipment, 2011, 3 (4): 55-58.

[2] Cui Jian, Li Jia.: Beijing: Machinery Industry Press, 2006

[3] Qin Changgui. The development and innovation of the design of [J]. electric control system of mechanical and electrical products combination machine tool based on PLC. 2011 (03).

[4] Zhao Liming, Li Junxiu. Application of programmable logic controller [M]. Chemical Industry Press, 2002

[5] Bian Chunyuan, President of Man Yongkui S7-300 /400. Shuangyan, PLC application development guide. Beijing: Mechanical Industry Press, 2007

[6] Jiang Pingping. Automation of manufacturing process automation control and detection 
technology [J]. manufacturing automation, 34, 2012 (9): 41-43. 